\title{
Experimental Study on Cooling Efficiency of Hypersonic Optical Window
}

\author{
Q. Mi, S. H. Yi ${ }^{\dagger}$, X. H. Zhao, B. Zhang and X. G. Lu \\ College of Aerospace Science and Engineering, National University of Defense Technology, No. 109, Deya \\ Road, Changsha, Hunan, 410073, China
}

†Corresponding Author Email: miqi2030@163.com

(Received January 20, 2021; accepted August 3, 2021)

\begin{abstract}
The cooling effectiveness of optical window influences the imaging quality of hypersonic vehicles. This study focuses on the supersonic film cooling efficiency on the optical window of a blunt cone in hypersonic flow. The experiments were conducted in a gun tunnel equipped with a Mach 8 nozzle providing a total pressure and temperature of $9 \mathrm{MPa}$ and $900 \mathrm{~K}$ respectively. Three tangential 2D nozzles with different combinations of slot heights and Mach numbers were designed to detect the film cooling length under different injection pressures. The heat flux on window surface was measured by Thin Film Gauges and the flow field was monitored by schlieren technique. When the jet pressure matched the mainstream, the window was completely cooled, and the minimum mass flow rate was achieved when the slot height was $5 \mathrm{~mm}$ and the jet Mach number was 2.5. If the pressure ratio of jet continues to increase, the heat flux density could be furtherly reduced, but the cooling efficiency of unit coolant mass flow decreased significantly. The data correlation results showed that the cooling efficiency presented a nonlinear relationship of second order polynomial with $\left(x / S_{h}\right) \lambda^{-0.8}$, and the effective cooling length of film was positively correlated with the cooling mass flow ratio $(\lambda)$ and slot height of the nozzle. Besides, the increase of jet pressure resulted in thickening the mixing layer, which enhanced the heat insulation effect and reduced the heat flux as a result.
\end{abstract}

Keywords: Optical window; Supersonic film; Injection nozzles; Cooling efficiency.

\section{NOMENCLATURE}

$M_{\infty} \quad$ Mach number of wind tunnel

$T_{0} \quad$ total temperature of wind tunnel

$P_{0} \quad$ total pressure of wind tunnel

$R_{\infty} \quad$ unit Reynolds number of mainstreams

$t \quad$ running time of wind tunnel

$l_{t} \quad$ lip thickness of nozzle

$S_{h} \quad$ slot height of nozzle

$A_{e} \quad$ area of nozzle exit

BFS Backward-Facing Step

$h \quad$ height of backward-facing step

$M_{j} \quad$ Mach number of jet

PRJ pressure ratio of jet

$T_{\text {ref }} \quad$ reference temperature

$T \quad$ measured temperature

$T_{w} \quad$ wall temperature

$\Delta T \quad$ temperature variation

$\rho \quad$ density of platinum film

$\alpha_{R} \quad$ temperature - resistance coefficient

$c$ heat $\delta$ boundary layer thickness heat conductivity half cone angle angle of attack heat flux lip thickness slot height Stanton number mass flow rate of jet density of mainstream velocity of mainstream density of jet velocity of jet cooling mass flow ratio heat flux under film injection condition heat flux under no injection condition cooling efficiency of film injection cooling efficiency of unit mass flow effective cooling length 


\section{INTRODUCTION}

The development of hypersonic vehicles (Ma>5) has brought new challenge to thermal protection from aerodynamic heating. In the flight test of the X-15 program, the outer windshield glass cracked due to the great temperature gradient induced by aerodynamic heating (Kelly et al. 1993). Although the thermal shield can effectively insulate the surface thermal load, but its surface ablation changes the flight performance and this problem worsens when flying at a higher speed continuously. Thus, a thermal protection system (TPS) combining both passive and active cooling methods need to be introduced on extremely heated components to limit structural temperature to acceptable level, such as the film cooling applied for gas turbine thermal protection and active regenerative cooling used in rocket engines (Zhang et al. 2020). For imaging guided vehicles, the surface thermal loads of its optical window profoundly affect the guidance precision. On the one hand, the window heated by the hypersonic flow will generate a temperature gradient and thermal deformation, resulting in the non-uniform refractive index distribution, which will distort the target signal in the transmission process. On the other hand, if the temperature of window surface rises too high, its thermal radiation will enhance the background noise and reduce the signalto-noise ratio of imaging (Zhang et al. 2014; Yin 2003). Therefore, it is necessary to protect the optical window by film cooling, but the payload and space limitations of the aircraft put forward higher requirements for cooling efficiency. The reasonable design of injection nozzles structure makes it possible to achieve the required cooling effect under the condition of as small flow as possible, which has engineering application value.

As an active thermal protection system, film cooling adopts low temperature gas injected from slot or orifices to form a protective film, thus separating the wall surface from the high temperature region within the boundary layer of incoming flow. Among various film cooling schemes, two-dimensional tangential injection has been widely studied due to its simple structure and good cooling effect. At first, researches aimed at subsonic jet. Stollery and El-ehwany (1965) put up with a subsonic film cooling model that divided the flow field into potential core region, wall jet region and boundary layer region. However, Aupoix et al. (1998) validated that the supersonic film was more efficient than the subsonic film by analyzing the Mach number profiles, because the supersonic injection could form a steady cold thin boundary layer before merging with the main flow downstream, while the cold core region of the subsonic injection was quickly destroyed by the mainstream, and the turbulence scales were much smaller in supersonic mixing layer. Moreover, the formed supersonic mixing layer can be controlled by changing the density ratios and convective Mach number to restrain the mixing rate. Besides, Dellimore et al. (2010) found that the growth rate of compressible mixing layer was much lower than that of incompressible mixing layer, so the supersonic film can maintain a longer cold boundary layer and had a higher cooling effectiveness. Since then, researchers had carried out amount of experimental and numerical studies on the Mach number, mass flow rate, gas properties and injecting direction. Richards and Stollery (1977) tested the cooling effects of different gas media in the hypersonic gun wind tunnel and found that hydrogen performed the best. Juhany et al. (1994) changed the mass flow rate by adjusting Mach number within 1.2 2.2 and the total temperature and studied its influence on cooling efficiency under the condition of pressure matching. The results showed that there was a slight improvement of around $10 \%$ for cooling effectiveness when promoting the Mach number of cooled injection from 1.5 to 1.8 . Hombsch and Olivier (2013) and Konopka et al. (2010) explored the film cooling effect from the perspective of flow state through shockwave wind tunnel experiment and large eddy simulation, respectively. Hombsch focused on the influence of incoming flow turbulence intensity while Konopka cared more about the flow state of injection flow. The former found that film cooling in laminar mainstream flow was about 5 times more efficient than in turbulent flow. The numerical results of Konopka showed that the cooling effectiveness of the turbulent injection was $4.8 \%$ lower than that of the laminar injection at the position of $x / S_{h}=10$.

The objects of above researches were mostly 2D simplified models, and they took the combustion chamber walls, turbine blades, engine nozzles as research background and mainly focused on the cooling effect but had little requirement on the flow field structure. There were other studies focused on the cooling of imaging window of optical seeker. Hodge et al. (1993) studied the active cooling system of hypersonic optical window and used $\mathrm{NO}_{2} / \mathrm{N}_{2} \mathrm{O}_{4}$ reactive coolant, and he defined a "breakpoint" where the cooling efficiency dropped below one. The results showed that the breakpoint for $\mathrm{NO}_{2}$ was higher by over an order of magnitude than that of $\mathrm{CO}_{2}$ or $\mathrm{N}_{2}$. Meanwhile, he also confirmed that the tangential jet had a higher cooling efficiency than jet with other injection angles. Fu et al. (2014) carried out an experiment of air film cooling on a 3D blunt body, and the results showed that increasing the blowing ratio could significantly improve the cooling effectiveness and prolong the cooling length, and a greater amount of cooling gas was required at a bigger angle of attack.

This study focused on the film cooling of optical imaging window of hypersonic guided vehicles. According to the typical optical dome configuration, a blunt cone with side window and three different kinds of two-dimensional tangential injection nozzles were designed. In this paper, platinum thin film gauges were used to measure the heat flux distribution on the window surface, combined with schlieren system to manifest the flow field structure above the window area. The gas film cooling effectiveness under different slot heights, Mach number of jets, mass flow rates and pressure ratios were investigated to explore the best cooling solution, which provided the basis for the design of cooling jet mechanism of optical flat window. 


\section{EXPERIMENTAL SETUP}

\subsection{KD-01 Hypersonic Gun Tunnel}

The experiments were conducted in the KD-01 hypersonic gun tunnel (Zhou et al. 2011) of National University of Defense Technology. The tunnel is 43 meters in length and mainly consists of high-pressure driving section, low-pressure driven section, axisymmetric nozzle, experimental chamber, and vacuum tank. The exit diameter of the nozzle is $500 \mathrm{~mm}$, and different throats can be replaced to adjust the Mach number from 7 to 10 . The tunnel works at a free piston driven mode, and it can be furtherly sorted into air driving mode and hydrogennitrogen driving mode according to different driving gas. The latter can provide a hypersonic flow with a higher total temperature at the same operating pressure. The experiments in this paper were carried out at the air driving mode, which produced a Mach 8 flow at a total temperature of $900 \mathrm{~K}$ and a total pressure of $9 \mathrm{MPa}$. The stable operation time of the tunnel is about $20 \mathrm{~ms}$. The specific parameters of the wind tunnel are shown in Table 1.

\subsection{Experimental Model}

The model of the optical dome adopts a blunt cone configuration, with a spherical head radius of $30 \mathrm{~mm}$. The half-cone angle $\theta$ is $13^{\circ}$, as marked in Fig. 1. A $13^{\circ}$ tangent plane on the upper side of the model is used to install the optical window, adjacent to the tangential injection nozzle inside a backward-facing step (BFS) configuration. The total length of the window is $328 \mathrm{~mm}$. The window is made of acrylic material and its thickness is $10 \mathrm{~mm}$, which can reduce the quick loss of surface heat flux and insulate to the thin film gauges (TFGs) to avoid transverse heat transfer, so that the heat flux measurement meets the one-dimensional semi-infinite hypothesis. The twodimensional tangential half Laval nozzle in BFS is $182 \mathrm{~mm}$ from the stagnation point of the head and $110 \mathrm{~mm}$ in width. The characteristic line method (Yi et al. 2013) is adopted to design the three nozzles with different slot heights and Mach numbers. To simplify the expression, the nozzle was named with its slot height and Mach number as "H_M_" (H represents slot height, $\mathrm{M}$ represents Mach number), and their specific parameters are displayed in Table 2 and Fig. 2. The Dry and clean nitrogen is used as the cooling gas with a total temperature of $300 \mathrm{~K}$.

Table 1 Parameters of KD-01 hypersonic gun wind tunnel

\begin{tabular}{|c|c|c|c|c|}
\hline$M_{\infty}$ & $T_{0} / \mathrm{K}$ & $P o / \mathrm{MPa}$ & $R e_{\infty} / 10^{6} \mathrm{~m}^{-1}$ & $t / \mathrm{ms}$ \\
\hline 8 & 900 & 9 & 15.0 & 20 \\
\hline
\end{tabular}

Table 2 Parameters of jet nozzles

\begin{tabular}{|c|c|c|c|c|}
\hline Nozzles & $l_{t} / \mathrm{mm}$ & $S_{h} / \mathrm{mm}$ & $A_{e} / \mathrm{mm}^{2}$ & $M_{j}$ \\
\hline H5M3 & 1 & 5 & 550 & 3 \\
\hline H4M3 & 2 & 4 & 440 & 3 \\
\hline H5M2.5 & 1 & 5 & 550 & 2.5 \\
\hline
\end{tabular}

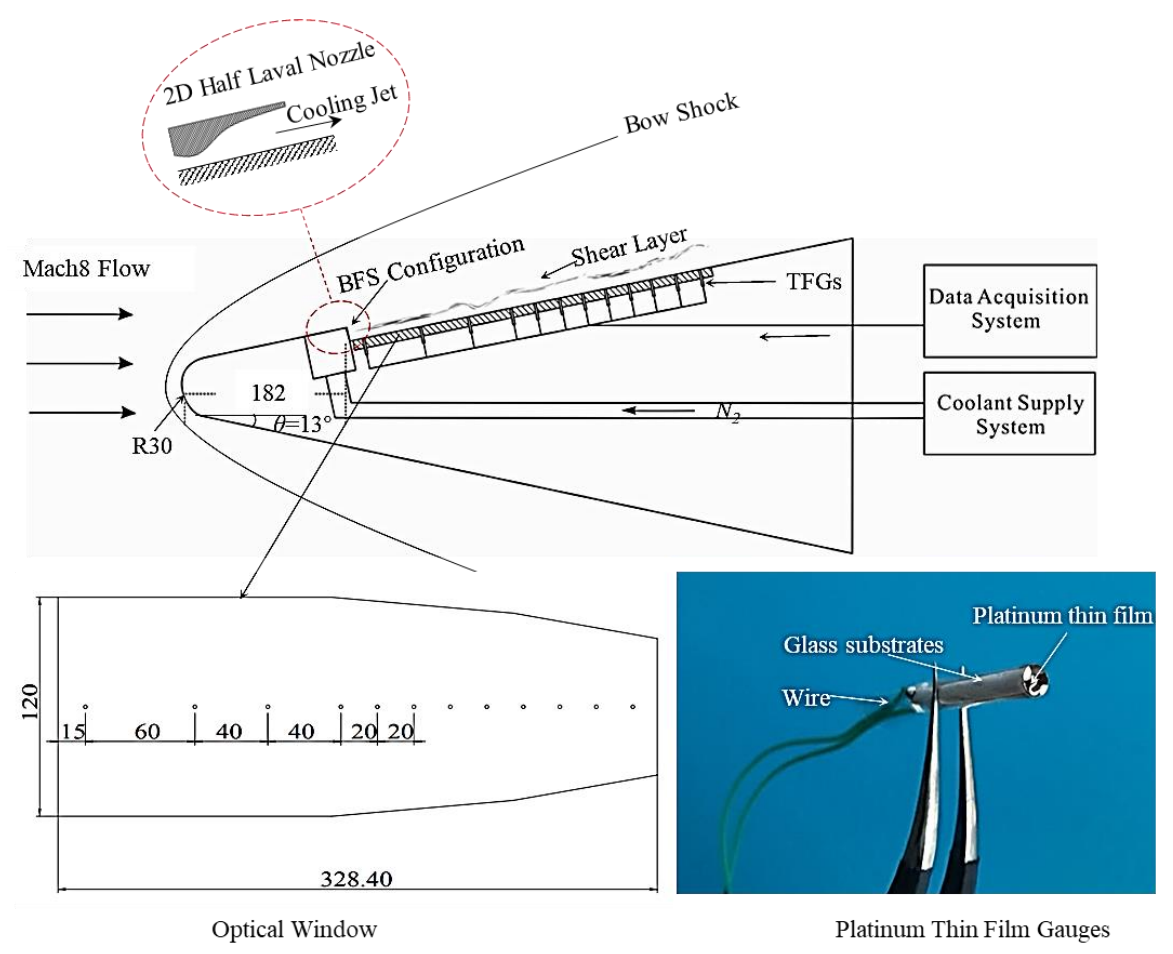

Fig. 1. Schematic of experimental setup. 


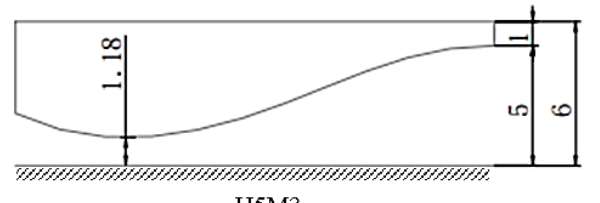

H5M3
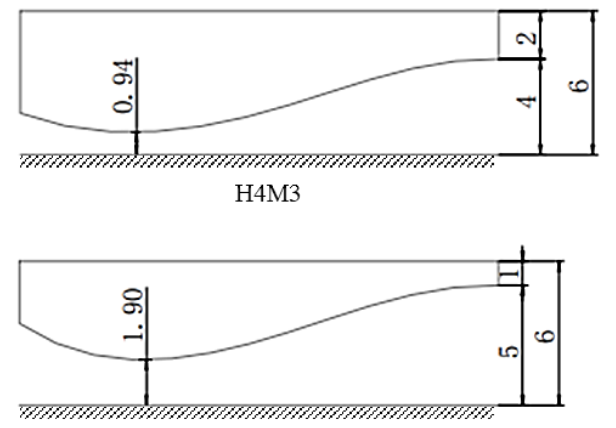

H5M 2.5

Fig. 2. Profiles of the three half-Laval nozzles.

\subsection{Thin Film Gauges (TFGs) for Heat Flux Measurement}

Thin Film Gauges (TFGs) was used to measure the heat flux distribution on window surface, and there were twelve TFGs assigned on the centerline of the optical window, shown as Fig.1. Since we focused on the cooling length of gas film, the distribution of gauging points is set as sparse in front part and dense in rear part, and the first gauge is $15 \mathrm{~mm}$ away from the leading edge of the window.

TFGs is widely adopted in transient thermal measurement due to its high sensitivity, fast response, and good linearity (Lu and Kinnear 1999). The TFG is composed of a "s" shaped platinum plating film with thickness of $100 \mu \mathrm{m}$, a glass cylinder base with $2 \mathrm{~mm}$ in diameter and $15 \mathrm{~mm}$ in length and wires, as shown in Fig.1. A constant current power supply is used to provide $20 \mathrm{~mA}$ current for each TFG. When the platinum film exchanges heat with the incoming flow, its resistance changes, thus, a varied voltage signal is collected. Its temperature-voltage linear relationship can be described as follows:

$\Delta T=T-T_{0}=\frac{\Delta R}{\alpha_{R} R_{0}}=\frac{\Delta V}{\alpha_{R} V_{0}}$

Among which, $T_{\text {ref }}$ is the reference temperature of the window surface, which was measured at the beginning of the experiments. $V_{0}$ is the initial voltage of TFGs, $\Delta V$ is the voltage variation during the experiment, and $\alpha_{R}$ is the temperature-resistance coefficient of the platinum film. The $\alpha_{R}$ of each TFG is different and needs to be calibrated in the thermostat before the experiment. Considering the actual temperature variation range of the model, 10 points within $10 \sim 30^{\circ} \mathrm{C}$ were selected to calibrate $\alpha_{R}$ by successive heating and cooling. Since the erosion of the high-speed flow may permanently changes $\alpha_{R}$ of the platinum film, the TFG needs to be recalibrated after several experiments. In previous study (Zhao et al. 2020), the surface temperature variation $\Delta T$ measured before and after recalibration by the TFGs, which had worked for 15 experiments, showed an error of less than $5 \%$.

$\Delta T$ is converted from the measured voltage signal and then the heat flux is calculated based on the onedimensional semi-infinite heat transfer theory:

$$
q_{\mathrm{w}}=\sqrt{\frac{\rho c k}{\pi}}\left[\frac{\Delta T_{\mathrm{w}}(t)}{\sqrt{t}}+\frac{1}{2} \int_{0}^{t} \frac{\Delta T_{\mathrm{w}}(t)-\Delta T_{\mathrm{w}}(\tau)}{(t-\tau)^{3 / 2}} \mathrm{~d} \tau\right]
$$

In the formula (2), $\rho, c, \kappa$ represent the density, specific heat, and heat conductivity of platinum film, respectively. $T_{w}$ is the surface temperature during the testing period, and $t$ is the effective test time. The integral term in the equation can be discretized by piecewise linear interpolation method ( $\mathrm{Fu}$ et al. 2014), then it can be converted into the following form:

$$
q_{\mathrm{w}}=\sqrt{\frac{\rho c k}{\pi}}\left\{\frac{\Delta T_{\mathrm{w}}(t)}{\sqrt{t}}+\sum_{i=1}^{n-1} \frac{\Delta T_{\mathrm{w}}\left(t_{i+1}\right)-\Delta T_{\mathrm{w}}\left(t_{i}\right)}{\left(t_{n}-t_{i+1}\right)^{1 / 2}-\left(t_{n}-t_{i}\right)^{1 / 2}}\right\}
$$

\section{RESULTS AND DISCUSSION}

\subsection{Heat Flux Measurement}

The ratio of static pressure at nozzle exit to that of mainstream above is defined as Pressure Ratio of Jet $\left(P R J=P_{e} / P_{\infty}\right)$, which is an important factor that influence the development of the cold boundary layer formed by cooling film, and it has great impact on the wave structure, thickness of mixing layer and the turbulence scale of cooling film (Zhang et al. 2019). The PRJ was controlled by changing the total pressure in the cooling jet chamber. According to the measured total pressure and total temperature in jet chamber, the mass flow rate of nitrogen in the nozzle was calculated out as Table 3 . The three nozzles were tested under the pressure conditions of no jet injection $(\mathrm{PRJ}=0)$, under-expanded jet $(\mathrm{PRJ}=0.5)$, pressure-matched jet $(\mathrm{PRJ}=1.0)$ and over-expanded jet $(\mathrm{PRJ}=1.5)$

Table 3 Experimental parameters of jets

\begin{tabular}{|c|c|c|c|}
\hline Nozzles & $P_{e} / \mathrm{kPa}$ & $\dot{m}_{j} /(\mathrm{g} / \mathrm{s})$ & $P R J$ \\
\hline \multirow{4}{*}{ H5M3 } & 0 & 0 & 0 \\
\cline { 2 - 4 } & 1.966 & 18 & 0.500 \\
\cline { 2 - 4 } & 4.047 & 37 & 1.008 \\
\cline { 2 - 4 } & 6.115 & 77 & 1.511 \\
\hline \multirow{4}{*}{ H4M3 } & 0 & 0 & 0 \\
\cline { 2 - 4 } & 1.971 & 13 & 0.503 \\
\cline { 2 - 4 } & 4.050 & 25 & 1.012 \\
\cline { 2 - 4 } & 6.011 & 44 & 1.497 \\
\hline \multirow{4}{*}{ H5M2.5 } & 0 & 0 & 0 \\
\cline { 2 - 4 } & 1.756 & 8 & 0.494 \\
\cline { 2 - 4 } & 4.046 & 19 & 1.019 \\
\cline { 2 - 4 } & 5.936 & 35 & 1.495 \\
\hline
\end{tabular}

According to the standard deviation of PRJ data in Table. 3, the control accuracy of pressure ratios was among $0.5 \% \sim 1 \%$. Since the experimental state 
cannot be the same in each experiment. It is necessary to derive the Stanton number (St) based on the measured heat flux as the non-dimensional heat transfer coefficient.

$$
S t=\frac{q_{w}}{\rho_{\infty} u_{\infty} c_{p}\left(T_{0}-T_{w}\right)}
$$

where $T_{0}$ represents total temperature of mainstream, and $T_{w}$ indicates the wall temperature. $\rho_{\infty}$ and $u_{\infty}$ are the density and velocity of local incoming flow, which can be obtained by the isentropic flow relation. Due to the extremely short running time of the gun wind tunnel, the wall temperature changes of window during the experimental period are relatively negligible, so the isothermal wall hypothesis is applicable to this study. Thus, the value of $T_{w}$ can be obtained as the initial wall temperature before the experiment. The corresponding heat transfer coefficient (St) were plotted as (a), (b) and (c) in Fig. 3 . The $x$ coordinate indicates the distance to the nozzle, which is nondimensionalized by the slot height of nozzle $\left(S_{h}\right)$. Meanwhile, to evaluate the uncertainty of measured data, we repeated some experiments for 3-5 times and plotted the error bar of each gauging point in Fig. 3(c). The statistical results showed that the average error percentage of gauging was around $4 \%$.

The curves with the square mark show the results of no injection condition, and we can observe a similar distribution along the flow direction: the Stanton number rises from the first point of about $5 \times 10^{-4}$, and climbs rapidly to the second point of about $1.2 \times 10^{-3}$, then decreases gradually. When the cooling film is introduced, the Stanton number drops dramatically. In the upstream part, the static temperature of the supersonic cooling film is much lower than the wall temperature, forming a negative temperature gradient. The wall conducts heat toward the coolant, making the surface heat transfer coefficient negative. When the Stanton number is 0 , it means there is no heat transferred from the external flow, that is, the window is completely protected from aerodynamic heating by incoming flow. Therefore, the interval with Stanton number less than 0 can be defined as the effective cooling length.

From Fig. 3(a) and Fig. 3(b), we can find that the window surface cannot be completely cooled when the jet Mach number is 3 and $P R J=0.5$, and the cooling length is about $45 S_{h}$ when the slot height is $5 \mathrm{~mm}$, and $50 S_{h}$ when the slot height is $4 \mathrm{~mm}$. Under this condition, the required mass flow rates of the two jets are $18 \mathrm{~g} / \mathrm{s}$ and $13 \mathrm{~g} / \mathrm{s}$ respectively. When the pressures of film injection and mainstream are matched $(P R J=1)$, both jets can achieve complete cooling for the window, but the mass flow rate consumed by the nozzle H4M3 is much smaller than that of nozzle H5M3. When the PRJ increases to 1.5, the heat transfer coefficient continues to drop, but the decrease is not significant while the coolant consumption almost doubles.

Comparing Fig. 3(a) and Fig. 3(c), when PRJ is 0.5 and slot height is $5 \mathrm{~mm}$, the cooling length of Mach 2.5 jet is shorter than that of Mach 3 jet. When

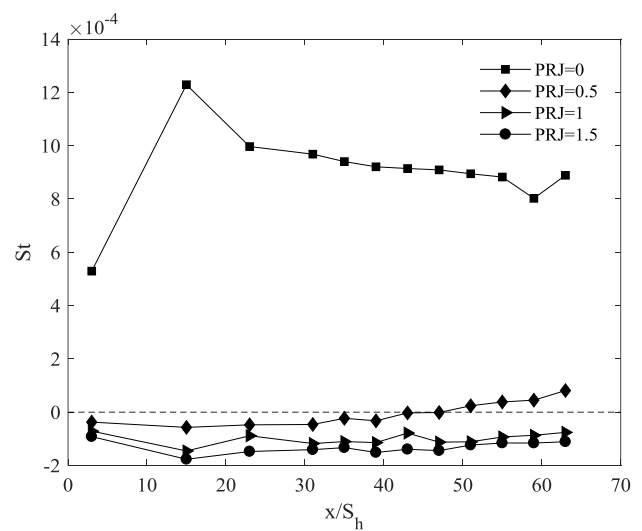

(a) $S_{h}=5 \mathrm{~mm}, \quad \mathrm{M}_{\mathrm{jet}}=3$

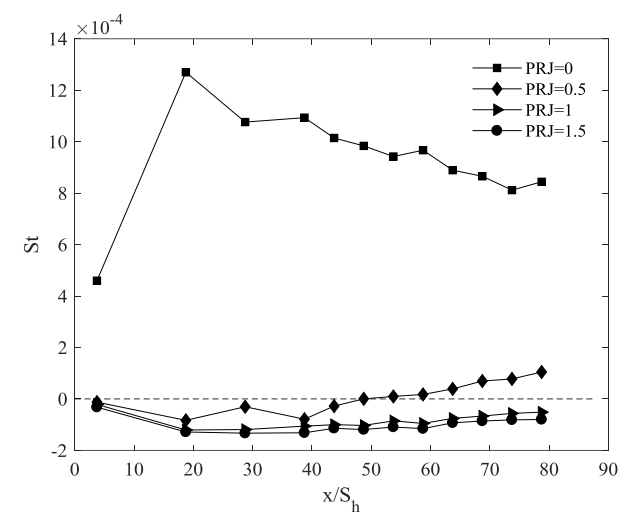

(b) $S_{h}=4 \mathrm{~mm}, \mathrm{M}_{\mathrm{jet}}=3$

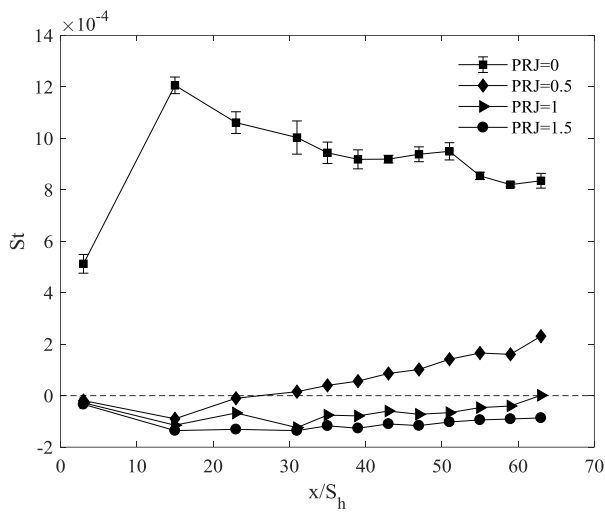

(c) $S_{h}=5 \mathrm{~mm}, \quad \mathrm{M}_{\mathrm{jet}}=2.5$

Fig. 3. Heat flux distribution under different cooling conditions.

$\mathrm{PRJ}=1$, Mach 2.5 jet can just completely cool the window, and the required mass flow rate is $19 \mathrm{~g} / \mathrm{s}$, which is only half of the consumption of Mach 3 jet at the same pressure ratio. When PRJ is adjusted to 1.5 , the mass flow rate consumed by the Mach 2.5 jet increases to $35 \mathrm{~g} / \mathrm{s}$, which is still much lower than the other two kinds of jets under the same pressure conditions.

According to the research conclusions of Goldstein et al. (1966) and Fu et al. (2014), the cooling mass flow ratio (blowing ratio, $\lambda$ ) is an important parameter that influences the film cooling effectiveness, and its definition is as follows: 
$\lambda=\frac{\rho_{j} u_{j}}{\rho_{\infty} u_{\infty}}$

$\rho_{j}$ and $u_{j}$ represent the density and velocity of jet flow respectively, while $\rho_{\infty}$ and $u_{\infty}$ indicate the density and velocity of the local mainstream. Since the experiments in this paper were carried out under the same incoming flow conditions, $\rho_{\infty} u_{\infty}$ is almost the same. Thus, changing the Mach number of jets changes $\rho_{j} u_{j}$, and changing the slot height changes the area of nozzle exit $A_{e}$, so the main factor that impacts the film cooling effectiveness is the mass flow rate.

$\dot{m}_{j}=\rho_{j} u_{j} A_{e}$

Bass et al. (1990) gave a definition of cooling efficiency of film injection based on the hypothesis of isothermal wall in his research:

$\eta=1-\frac{Q_{j}}{Q_{0}}$

Among which, $Q_{0}$ represents the surface heat flux density with no jet injection, and $Q_{j}$ represents the heat flux density when the film jet is introduced.

According to the results shown in Fig. 3, when the jet pressure is matched, all the three nozzles can completely cool the window. To accurately evaluate the cooling efficiency of the unit mass flow of the three nozzles under this state, we defined a cooling coefficient $C_{m_{i}}$ associated with the mass flow rate, and the bigger cooling coefficient means the higher cooling efficiency of the unit mass flow. The results were presented as Fig. 4. To compare the actual cooling length uniformly, the $x$ coordinate was nondimensionalized by the height of backwardfacing step $(h)$.

$C_{\dot{m}_{j}}=\frac{\eta}{\dot{m}_{j}}$

Based on the repeated experimental data, the measurement errors of heat flux and mass flow rate were acquired, and we plotted the error bar of cooling coefficient of H5M2.5 nozzle under the condition of $\mathrm{PRJ}=1$. The average error percentage for the calculation of the cooling coefficient was

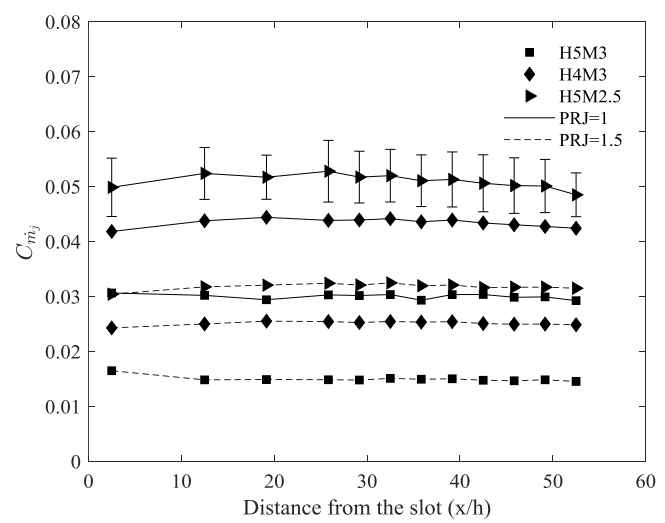

Fig. 4. Cooling coefficient of unit mass flow of the three nozzles. around $10 \%$. According to Fig. 4, the H5M2.5 nozzle achieved the highest cooling efficiency of unit mass flow, while the H5M3 nozzle had the lowest cooling coefficient. Contrasting the solid curves with dashed ones, we can find that, although the heat flux can be furtherly reduced at the condition of over-pressure (PRJ>1), but its cooling efficiency of unit mass flow dramatically dropped, which means a greater demand of coolant amount to achieve the same cooling purpose in flight. In the case of pressure matched, the film cooling length can not only totally cover the window area, but also its cooling efficiency of unit mass flow rate is relatively high. Therefore, jet pressure matching $(\mathrm{PRJ}=1)$ is a more appropriate choice.

Furtherly, we synthesized the injection parameters to acquire the universal law. Based on the research of Bass et al. (1990), Cary and Hefner (1972), and Parthasarathy et al. (1970), we plotted the film cooling efficiency against the dimensionless parameter of $\left(x / S_{h}\right) \cdot \lambda^{-0.8}$, which takes the cooling mass flow ratio and slot height into consideration comprehensively, and correlates the data in a relatively narrow band.

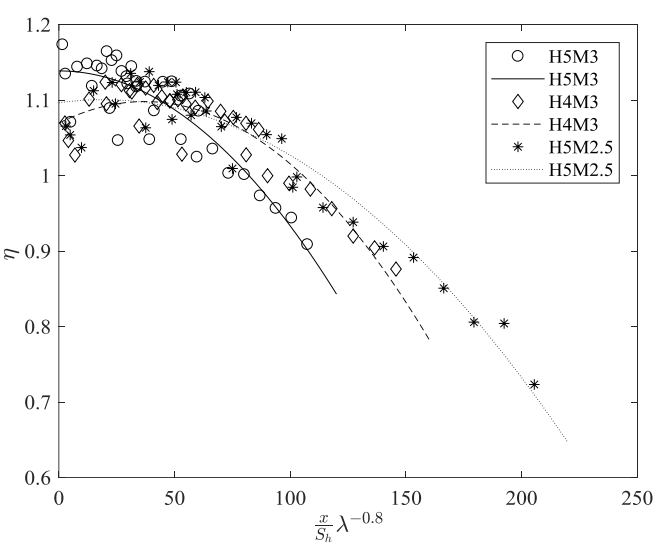

Fig. 5 Cooling efficiency data correlation.

According to the curve fitting tool, the cooling

efficiency shows a nonlinear correlation of second order polynomial with $\left(x / S_{h}\right) \cdot \lambda^{-0.8}$. To compare the performance of different nozzles, we have correlated the data of each nozzle and acquired their fitting curves, respectively, among which, solid curve indicates the cooling efficiency curve of the H5M3 nozzle. Its specific relation is:

$\eta=-2.05 \times 10^{-5}\left(\frac{x}{S_{h}} \lambda^{-0.8}\right)^{2}-1.11 \times 10^{-6}\left(\frac{x}{S_{h}} \lambda^{-0.8}\right)+1.1386$

thus, the effective cooling length $(\eta=1)$ can be derived as:

$X_{C L} / S_{h} \approx 82 \lambda^{0.8}$

Dashed curve represented that of the H4M3 nozzle, and its equation and solution are: 
$\eta=-2.08 \times 10^{-5}\left(\frac{x}{S_{h}} \lambda^{-0.8}\right)^{2}+0.0015\left(\frac{x}{S_{h}} \lambda^{-0.8}\right)+1.0694$

$X_{C L} / S_{h} \approx 106 \lambda^{0.8}$

While the correlation results of the H5M2.5 nozzle can be descripted as:

$\eta=-1.13 \times 10^{-5}\left(\frac{x}{S_{h}} \lambda^{-0.8}\right)^{2}+0.00043\left(\frac{x}{S_{h}} \lambda^{-0.8}\right)+1.0964$

$X_{C L} / S_{h} \approx 114 \lambda^{0.8}$

Contrasting the equation (12), (14) and (16), we can concluded that the H5M2.5 nozzle has the best performance in film cooling, since it consumes the least coolant to achieve a certain effective cooling length under the same incoming flow conditions.

In this study, the effective cooling length demanded for optical window is $328 \mathrm{~mm}$, about $66 S_{h}$ when using the H5M2.5 nozzle, and its required cooling mass flow ratio $(\lambda)$ is about 0.5 when the $P R J=1$, which is in accordance with the test results in Fig. 3(c). A higher $\lambda$ would get a better cooling effect of window aera but lead to a waste of coolant.

\subsection{Flow Visualization}

To explore the flow mechanism of film cooling, Nano-tracer-based Planar Laser Scattering (NPLS) and schlieren techniques was applied to monitor the flow structures across the window under different jet pressures of nozzle H5M2.5. The NPLS technology is a noncontact fine flow structure visualization technique with high temporal and spatial resolution, which is originally created by the author's research group (Zhao et al. 2009). The NPLS system employs a laser sheet to illuminate a flow field containing evenly dispersed nanoparticles. The Rayleigh scattering of nanoscale tracer particles lighted by laser sheet establishes the relationship between image gray level and flow field density. Thus, the outline of the boundary layer can be distinguished due to the great density gradient. It has been proved to be mature and reliable by successfully measuring the coherent structures in supersonic boundary layer (He et al. 2011), density field of supersonic mixing layer, shockwave and turbulent boundary layer interactions ( $\mathrm{Lu}$ et al. 2020) and hypersonic boundary layer (Yi et al. 2020). More information about the NPLS system composition and work principles can be referred from the above literatures.

Thus, in the same incoming flow, the boundary layer across the nozzle exit had no significant change when there was no cooling injection. From the Fig. 6 , we can observe the bow shock and the weak reattachment shock. The boundary layer upstream the nozzle exit remained in the laminar state. According to the binarization processing result of the zone in red dashed rectangle, the incoming boundary layer thickness $(\delta)$ was almost equal to the height of the BFS, that was $\delta / h \approx 1$.

Since the wave structures are so weak to be captured in such a slice of laser. Therefore, it is necessary to

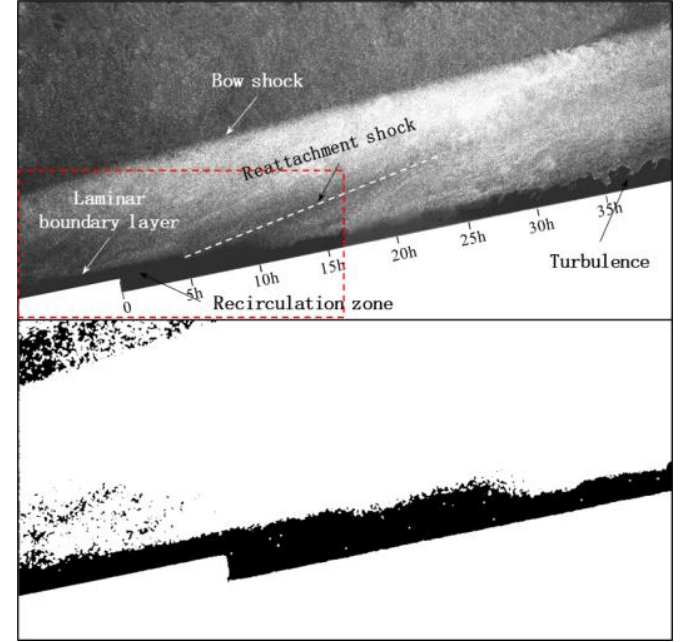

Fig. 6. boundary layer images under no cooling condition.

adopt traditional schlieren system to show the compression and expansion waves. The knife edge cuts from bottom to top. Figure 7(a) shows the no injection scenario, the flow structure is similar to the typical hypersonic flow over the BFS (Chen et al. 2018), that is, the incoming flow is blocked by the blunt head of the model and form a bow shock. Then, an expansion fan occurs when the flow crosses the step corner at the jet nozzle exit. The mainstream reattaches to the window surface at about $4 h$ and generate a reattachment shock with an angle of $23.7^{\circ}$, which corresponded to the NPLS image.

When PRJ is 0.5 , the flow structure become more complicated, as shown in Fig. 7(b). The weak compression wave shown as "Shockwave I" in the picture is induced by the installation seam of jet nozzle. A weak shock wave (shown by the yellow dotted line) appears when main flow encounters the film jet at upper tip of the nozzle lip, and its angle is about $28.2^{\circ}$. This is a characteristic of supersonic film jet (Meinke et al. 2012), At this time, due to under-expand flow state of the jet, part of the expansion waves can still be observed. Downstream of the nozzle exit, multiple weak compression waves are captured, but it is difficult to analyze their origins because of the unclear structure. Since the jet pressure is lower the than mainstream, the mixing layer formed by the interaction between cooling film and the mainstream is inclined to the wall.

When the jet pressure matches the mainstream $(P R J=1)$, a variety of wave structures can be clearly observed in Fig. 7(c). Firstly, the incoming flow is compressed by the jet at the upper tip of the lip and an upper lip shock with an angle of about $30.5^{\circ}$ was produced. Then, at the lower tip of the lip, it mixes and shears with the jet flow, forming a mixing layer. The upper edge of the mixing layer is initially parallel to the wall. Then, the mixing layer thickens so quickly that the jet is compressed by its lower edge and produces a lower lip shock wave. The lower lip shock wave is reflected on the wall and passes through the mixing layer, propagating 


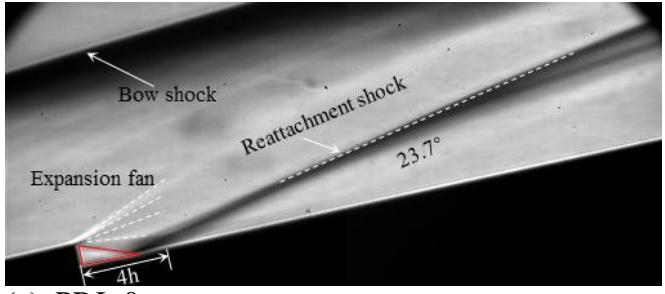

(a) $\mathrm{PRJ}=0$

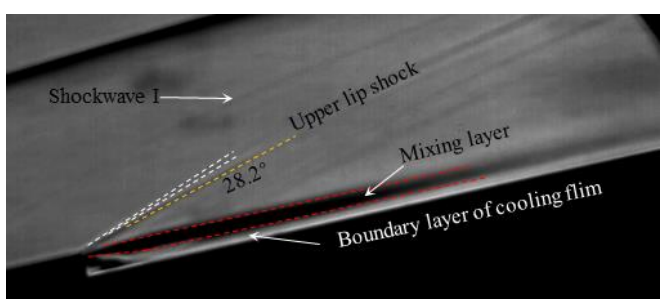

(b) $\mathrm{PRJ}=0.5$

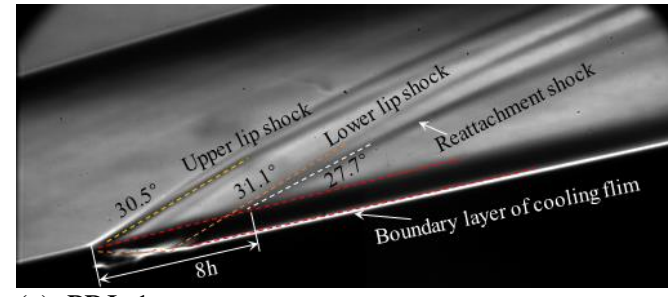

(c) $\mathrm{PRJ}=1$

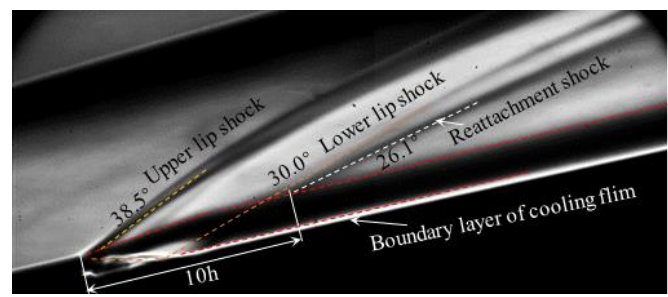

(d) $P R J=1.5$

Fig. 7. Schlieren images under different pressure ratios of jet ejected by nozzle H5M2.5.

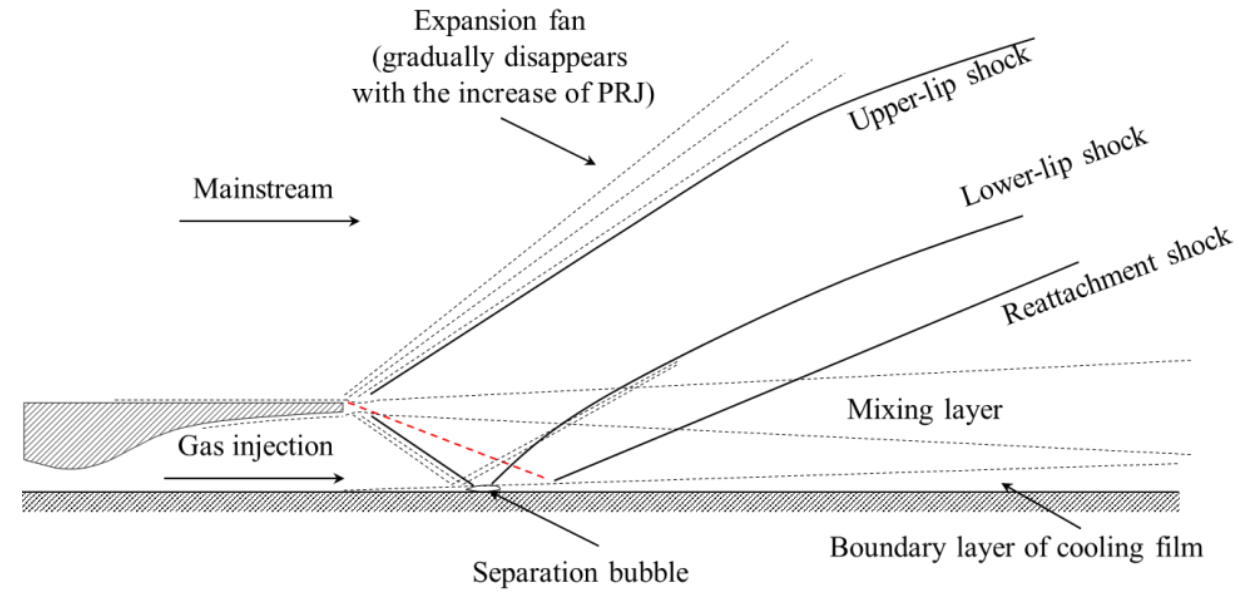

Fig. 8. Simplified flow structure of film cooling on window surface.

downstream (shown by the orange dashed line) with the angle of about $31.1^{\circ}$. The mixing layer continues to develop and attaches to the wall at about $8 \mathrm{~h}$ from the nozzle exit and generates a reattachment shock (shown by the white dashed line) with the angle of about $27.7^{\circ}$.

When PRJ is promoted to 1.5 , the basic flow structure changes little. The only differences are that the angle of upper lip shock increases to $38.5^{\circ}$ while the angle of lower lip shock reduces to $30.0^{\circ}$, and the reattachment length of mixing layer extends to $10 \mathrm{~h}$ but the angle of reattachment shock declines to $26.1^{\circ}$.

By comparing Fig. 7(b), (c), (d), it can be clearly observed that, as PRJ continues to increase, the expansion fan disappears gradually, and the compression effect of the film jet on the main flow strengthens, behaving as the inclination angle of the upper-lip shock keeps increasing and the development direction of the mixing layer gradually turns to the mainstream. Moreover, the reattachment position of mixing layer tends to delay, and its thickness keeps increasing, from the average thickness of $0.8 h$ at $\mathrm{PRJ}=0.5$ to $1.1 h$ at the matched condition. When PRJ=1.5, the average thickness of the mixing layer reaches $1.4 h$.

From the views in Fig. 7, the flow field structure of the blunt body in Mach 8 flow with the window surface covered by the supersonic gas film resembles the film cooling structure on plate in the Mach 2.95 flow of Song and Shen (2018). The flow field in this study can be simplified to the general structure shown in Fig. 8.

Combining with the schlieren images in Fig. 7, we can primarily explain the heat transfer characteristics in Fig. 3. Since the first gauging point is only about $2 h$ away from the nozzle exit, it is completely in the recirculation zone (the area shown by the red triangle in Fig. 7(a)), in which the flow velocity and thermal conductivity are very low, so the heat flux density is the lowest. Then, the flow reattaches at about $4 h$ away from the step corner. According to the research conclusions of Reddeppa et al. (2011), the heat flux 
at the reattachment point will reach a peak value higher than the recirculation zone, after which the heat flux gradually decreases. Because the second measuring point is closest to the reattachment position, the heat flux density is higher than other points. Besides, it can be found that with the increase of pressure ratio of jet, the demand for the coolant mass flow rate rises sharply, which is reflected in the thickening of the mixing layer in the flow field. As a result, the heat insulation effect of cooling film is enhanced, and the heat flux density reduces overall.

\section{Conclusion}

This study explored the cooling efficiency of three jet nozzles and the effects of pressure ratio of jet (PRJ) on the optical window of a blunt cone in Mach 8 flow. The heat flux distribution on window surface was measured by the Thin Film Gauges and the flow structure was visualized by Schlieren technique.

Based on the heat-transfer distributions, when the jet pressure was matched, the three nozzles could completely cool the window, among which the nozzle H5M2.5 demanded the least coolant and had the highest cooling efficiency of unit cooling mass flow. When PRJ increased to 1.5, the heat flux density can be reduced furtherly, but the cooling efficiency of unit coolant dropped dramatically.

By data correlations between dimensionless parameters of jet flow and cooling efficiency, it was found that the cooling efficiency presented a nonlinear relationship of second order polynomial with $\left(x / S_{h}\right) \lambda^{-0.8}$, and the effective cooling length of film was positively correlated with the cooling mass flow ratio $(\lambda)$ and slot height of the nozzle. Significantly, the H5M2.5 nozzle had the best cooling effectiveness according to the correlation results. For the complete cooling of optical window with the length of about $66 S_{h}$, the demanded coolant mass flow ratio $(\lambda)$ is 0.5 at $P R J=1$ when employing the H5M2.5 nozzle.

According to schlieren images, the increase of the mass flow rate of film injection led to a stronger compression effect of the cooling film to the mainstream and a thicker mixing layer, which enhanced the thermal insulation effect between wall and external flow, thereby reducing the heat flux density.

\section{ACKNOWLEDGEMENTS}

This work was supported by the National Key Research and Development Plan of China (Grant No. 2019YFA0405300) and the National Project for Research and Development of Major Scientific Instruments of China (Grant No. 11527802) and the Major Research Plan of the National Natural Science Foundation of China (Grant No. 91752102 and No. 11832018). This support is gratefully acknowledged.

\section{REFERENCES}

Aupoix, B., A. Mignosi and S. Viala (1998). Experimental and Numerical Study of Supersonic Film Cooling. AIAA Journal 36(6), 915-923.
Bass, R., L. Hardin, R. Rodgers and R. Ernst (1990). Supersonic film cooling. In Proceedings of 2 nd International Aerospace Planes Conference: American Institute of Aeronautics and Astronautics. Orlando, U.S.A.

Cary, A. and J. Hefner (1972). Film-Cooling Effectiveness and Skin Friction in Hypersonic Turbulent Flow. AIAA Journal 10(9), 11881193.

Chen, L., K. Asai, T. Nonomura, G. Xi and T. Liu (BFS) flow mechanisms, heat transfer and control. Thermal Science and Engineering Progress 6, 194-216.

Dellimore, K. H., A. W. Marshall and C. P. Cadou (2010). Influence of Compressibility on FilmCooling Performance. Journal of Thermophysics and Heat Transfer 24(3), 506515.

Goldstein, R. J., E. R. G. Eckert, F. K. Tsou and A. Haji-Sheikh (1966). Film cooling with air and helium injection through a rearward-facing slot into a supersonic air flow. AIAA Journal 4(6), 981-985.

Fu, J., S. H. Yi, X. H. Wang, L. He and Y. Ge (2014). Experimental study on supersonic film cooling on the surface of a blunt body in hypersonic flow. Chinese Physics B 23(10), 104702.

He, L., S. H. Yi, Y. X. Zhao, L. F. Tian and Z. Chen (2011). Visualization of coherent structures in a supersonic flat-plate boundary layer. Chinese Science Bulletin 56(6), 489-494.

Hodge, R. A., P. Raghuraman and A. L. Murray (1993). Window cooling technology program. Journal of Spacecraft and Rockets. 30(4), 466476.

Hombsch, M. and H. Olivier (2013). Film cooling in laminar and turbulent supersonic flows. Journal of Spacecraft and Rockets 50(4), 742-753.

Juhany, K. A., M. L. Hunt and J. M. Sivo (1994). Influence of injectant Mach number and temperature on supersonic film cooling. Journal of Thermophysics and Heat Transfer 8(1), 59-67.

Kelly, H. N. and M. L. Blosser (1992). Active cooling from the sixties to NASP. Technical Report TM109079, NASA Langley Research Center, Hampton, USA.

Konopka, M., M. Meinke and W. Schröder (2011, April). Large-Eddy Simulation of Supersonic Film Cooling at Laminar and Turbulent Injection. In 17th AIAA International Space Planes and Hypersonic Systems and Technologies Conference, San Francisco, U.S.A.

Lu, F. K. and K. M. Kinnear (1999). Characterization of Thin-Film Heat-Flux Gauges. Journal of Thermophysics and Heat Transfer 13(4), 548549.

Lu, X. G., S. H. Yi, L. He, D. D. Gang and H. B. Niu 
(2020). Experimental Study on Unsteady Characteristics of Shock and Turbulent Boundary Layer Interactions. Fluid Dynamics 55(4), 566-577.

Meinke, M., W. Schroeder and M. Konopka (2012). Large-Eddy Simulation of Shock/Cooling-Film Interaction. American Institute of Aeronautics and Astronautics. 50(10), 2102-2114.

Parthasarathy, K. and V. Zakkay (1970). An experimental investigation of turbulent slot injection at Mach 6. AIAA Journal 8(7), 13021307.

Reddeppa, P., K. Nagashetty, S. Saravanan, G. Jagadeesh and S. L. Gai (2011). Measurement of Heat Transfer Rate on Backward-Facing Steps at Hypersonic Mach Number. Journal of Thermophysics and Heat Transfer 25(3), 321328.

Richards, B. and J. Stollery (1977, June). An experimental study of the cooling effectiveness of a laminar two-dimensional tangential film in hypersonic flow. In Proceedings of 10th Fluid and Plasmadynamics Conference, Albuquerque, U.S.A.

Song, C. Q. and C. B. Shen (2018). Effects of feeding pressures on the flow field structures of supersonic film cooling. Journal of Thermophysics and Heat Transfer 32(3), 648658.

Stollery, J. L. and A. El-ehwany (1965). A note on the use of a boundary-layer model for correlating film-cooling data. International Journal of Heat and Mass Transfer 8(1), 55-65.

Yi, S. H., Y. X. Zhao and L. He (2013). Supersonic and hypersonic nozzle design. National Defense Industry Press, Beijing, China.
Yi, S. H, X. L. Liu and X. G. Lu (2020) Application of NPLS technique in the researches on hypersonic boundary layer transition. Acta Aerodynamica Sinica 38(2), 348-354.

Yin, X. L. (2003). Principles of aero-optics. China Aerospace Publishing House, Beijing, China.

Zhang, F., S. H. Yi and Y. Y. Wu (2019). Ratio of static pressure influence on cooling film of hypersonic optical dome based on NPLS. Acta Aerodynamica Sinica 37(5), 762-769.

Zhang, T. X., H. Y. Hong and X. Y. Zhang (2014). Aero-Optical effect correction: principles, methods, and applications. University of Science and Technology of China Press, Hefei, China.

Zhang, S. L., X. Li, J. Y. Zuo, J. Qin and K. L. Cheng (2020). Research progress on active thermal protection for hypersonic vehicles. Progress in Aerospace Sciences 119, 376-421.

Zhao, X. H., S. H. Yi and F. Zhang (2020). Experimental study on the cooling film effectiveness of a hypersonic blunt body. Journal of Thermophysics and Heat Transfer (4), 1-4.

Zhao, Y. X., S. H. Yi, L. F. Tian and Z. Y. Cheng (2009). Supersonic flow imaging via nanoparticles. Science in China 52(12), 36403648 .

Zhou, Y. W., S. H. Yi, Z. Chen, X. G. Lu and Y. Ge (2011). KD-01 Hypersonic gun wind tunnel. Technical Achievement, National University of Defense Technology, Changsha, China. 